\title{
Standards and pitfalls of focal ischemia models in spontaneously hypertensive rats: With a systematic review of recent articles
}

Hiroshi Yao ${ }^{1 *}$ and Toru Nabika ${ }^{2}$

\begin{abstract}
We reviewed the early development of various focal ischemia models in spontaneously hypertensive rats (SHR), and summarized recent reports on this topic. Among 6 focal ischemia models established in divergent substrains of $\mathrm{SHR}$, distal middle cerebral artery occlusion is the most frequently used and relevant method of focal ischemia in the light of penumbra concept. We performed an online PubMed search (2001-2010), and identified 118 original articles with focal ischemia in SHR. Physiological parameters such as age, body weight, and even blood pressure were often neglected in the literature: the information regarding the physiological parameters of SHR is critical, and should be provided within the methodology section of all articles related to stroke models in SHR. Although the quality of recent studies on neuroprotective strategy is improving, the mechanisms underlying the protection should be more clearly recognized so as to facilitate the translation from animal studies to human stroke. To overcome the genetic heterogeneity in substrains of SHR, new approaches, such as a huge repository of genetic markers in rat strains and the congenic strategy, are currently in progress.
\end{abstract}

Keywords: Focal ischemia, Hypertension, Experimental, Genetics, Animal Models, Cerebrovascular disease, Stroke

\section{Introduction}

Almost 50 years have passed since the spontaneously hypertensive rats (SHR) were established [1]. In the present review, we will focus on the early development of focal ischemia models in SHR, and provide a critical systematic review on recent reports on SHR stroke models. SHR are one of the most widely used genetic models for hypertension. Hypertension is a major risk factor for stroke and most other cardiovascular diseases, and therefore SHR are relevant to stroke research. SHR were initially obtained by selective inbreedings from the Wistar-Kyoto rats (WKY) with the highest blood pressure. The stroke prone SHR (SHRSP) were established from the A substrain of the SHR, and the other 2 substrains $(\mathrm{B}$ and $\mathrm{C})$ are resistant to spontaneous stroke [2]. The B substrain corresponds to SHR/Izm (Izumo). SHR

* Correspondence: hyao@hizen2.hosp.go.jp

'Laboratory for Neurochemistry, Center for Emotional and Behavioral Disorders, National Hospital Organization Hizen Psychiatric Center, Mitsu 160, Yoshinogari, Kanzaki, Saga, 842-0192, Japan

Full list of author information is available at the end of the article were sent to the National Institute of Health $(\mathrm{NIH})$ at the F13 generation in 1966.

The advantages of using SHR in contrast to normotensive rats in stroke research are: (1) presence of comorbidity (i.e., hypertension), (2) reproducible and adequate-sized infarction after distal middle cerebral artery occlusion (MCAO) alone, and (3) a similar therapeutic time window and cerebral blood flow (CBF) threshold for infarction to normotensive rats [3,4]. Shortcomings are: (1) SHR and SHRSP are expensive, (2) high mortality in aged SHR and SHRSP, and (3) resistance to therapy. Another confounding problem with using SHR in stroke research is that SHR and WKY from different sources are genetically heterogeneous [5-7]. Because WKY are rarely used for focal ischemia models or their controls in recent years, we do not mention detailed descriptions of genetic heterogeneity of WKY.

\section{Stroke proneness and stroke sensitivity}

Focal ischemia models are usually based on MCAO and mimic human acute brain infarction caused by occlusion of major cerebral arteries. Small vessel disease is another

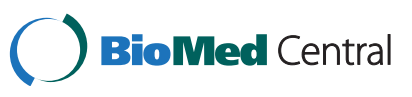

(c) 2012 Yao and Nabika; licensee BioMed Central Ltd. This is an Open Access article distributed under the terms of the Creative Commons Attribution License (http://creativecommons.org/licenses/by/2.0), which permits unrestricted use, distribution, and reproduction in any medium, provided the original work is properly cited. 
subtype of stroke, and is a major cause of vascular cognitive impairment. Spontaneous stroke in SHRSP, often under salt loading, is a unique and relevant feature of SHRSP. The most preponderant site for spontaneous stroke in SHRSP was the cortical area supplied by the anterior and posterior cerebral arteries with recurrent branching [8]. A systematic review summarized that animals sacrificed after developing stroke-like symptoms displayed arteriolar wall thickening, subcortical lesions, enlarged perivascular spaces and cortical infarcts and hemorrhages [9]. Hainsworth and Markus [10] concluded that to model small vessel disease-like arteriopathy, SHRSP appears closest to human small vessel disease, particularly in aged animals. In salt-loaded SHRSP, the number of days necessary to develop stroke has been used as an index of stroke proneness $[11,12]$. In contrast, infarct volume after MCAO is an index of stroke sensitivity. Tamura et al. described the first surgical MCAO in the rat [13]. Subsequently, the importance of hypertension was emphasized in focal ischemia models [14-16]. Both SHR and SHRSP have increased stroke sensitivity to focal ischemia (i.e., larger infarct size after MCAO) compared with normotensive rats [17]. Increased stroke sensitivity is likely attributable to not only spontaneous hypertension but also additional genetic factors that will be discussed later. Many neuroprotective strategies in stroke research are based on the penumbra concept constructed using focal ischemia models [18]. In this context, focal ischemia models are relevant to the human clinical setting of ischemic stroke or brain infarction.

\section{Focal ischemia models in SHR Distal MCAO}

Six focal ischemia models have been established in SHR and SHRSP (Figure 1). SHRSP maintained at the University of Michigan originated from the NIH stock in 1981 [19].

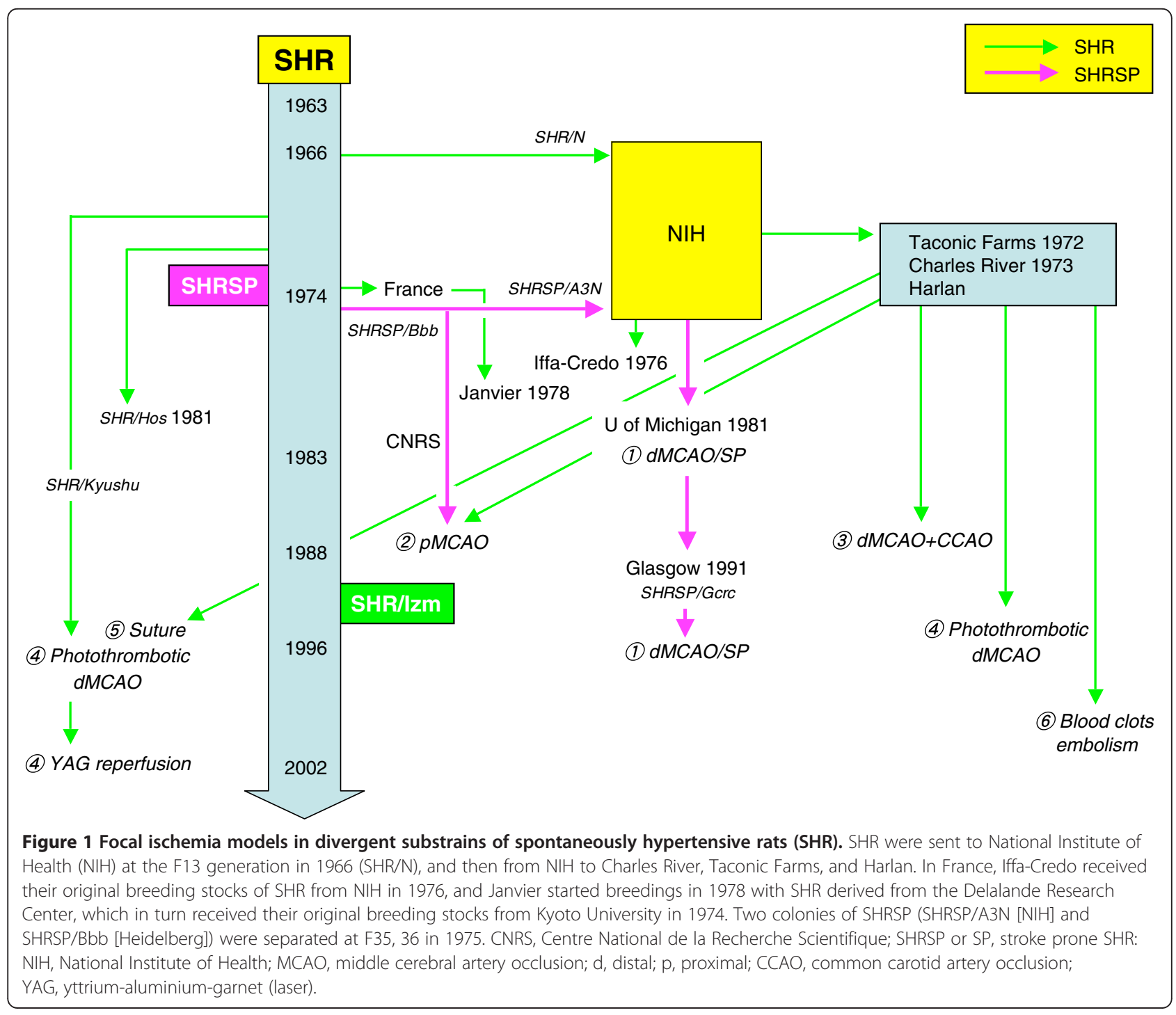


Using SHRSP/Michigan, the pathophysiological consequences of MCAO distal to striate branches were studied for the first time in SHRSP [14]. Even in young SHRSP without chronic hypertension, distal MCAO produced larger lesions than WKY or Sprague-Dawley rats. This approach of Coyle was later employed by Fujii et al. to investigate the beneficial effects of chronic antihypertensive treatment on infarct size after MCAO: systolic blood pressure in SHRSP was reduced to the level of WKY with antihypertensive treatment after 3 months of age for 3 months, which reduced infarct volume by approximately $40 \%$ compared with untreated SHRSP [20]. The inbred colonies of SHRSP, maintained in the Department of Medicine and Therapeutics at the University of Glasgow (SHRSPGla or SHRSP/Gcrc) since 1991, were derived from the colony of University of Michigan.

\section{Proximal MCAO}

The approach of Tamura et al., which occludes middle cerebral artery (MCA) proximal to the lenticulostriate arteries, produces infarction of both the cortex and the lateral part of striatum [13]. Duverger and MacKenzie employed the modified method of Tamura et al., using SHRSP (Centre National de la Recherche Scientifique [CNRS], France), SHR (Charles River, Italy), and 3 normotensive strains (WKY, Sprague-Dawley, and Fischer-344) [15]. In hypertensive rats, MCAO resulted in a considerable volume of infarction $(158.1 \pm 4.4$ [SEM] $\mathrm{mm}^{3}$ in SHR and $172.4 \pm 4.4$ [SEM] $\mathrm{mm}^{3}$ in SHRSP), and the variability was minimum (coefficient of variation $[$ C.V.] $=7-8 \%$ ) compared with the 3 normotensive strains (C.V. $=20-49 \%$ ). In the normotensive control WKY, the infarction was small $\left(63.2 \pm 10.9[\mathrm{SEM}] \mathrm{mm}^{3}\right)$.

\section{Tandem MCAO}

Brint et al. summarized their experience in 3 strains of rats (SHR/Charles River or Taconic Farms, Wistar, and Fisher-344 rats) with focal ischemia produced by tandem occlusion of the distal MCA and ipsilateral common carotid artery [16]. Of the 3 strains of rats, the SHR showed the largest $\left(157-259 \mathrm{~mm}^{3}\right)$ and most reproducible (average C.V. $=19 \%$ ) infarctions, and statistical power analysis revealed that tandem occlusion of the distal MCA and ipsilateral common carotid artery in the SHR strain offered a practical model in terms of requisite animals necessary to avoid a Type 2 error (false negative) for differences in infarct volume between control and experimental groups. Although infarct volume might be more reproducible in SHR with tandem carotid artery occlusion, the amount of penumbra tissue may also be reduced. Consequently, it may be more difficult to demonstrate neuroprotective efficacy in these models with tandem occlusion.

\section{Photothrombotic MCAO}

In the context of thrombosis, the effects of thrombotic stroke on compromised brain tissue may be different from those due to cerebral ischemia induced by mechanical occlusions of intracranial or extracranial brain arteries [21]. Prado et al. first used SHR as a photothrombotic distal MCAO model, avoiding common carotid artery involvement [22]. SHR/Kyushu and later SHR/Izm were employed for photothrombotic distal MCAO [23,24]. The SHR/Kyushu were from the F20 and F21 generations derived by Okamoto and Aoki [25]. A photothrombotic distal MCAO model in SHR yields a highly reproducible infarct volume (average C.V. $=21 \%$ ) and does not entail extensive surgery or opening of the dura, thereby avoiding unacceptable local tissue trauma at the site of MCAO [17]. This model encompasses appropriate physiological monitoring, associated risk factors for stroke, and clinically relevant pathophysiology of thrombosis. Cai et al. showed the infarct size was larger in male and female SHR/Kyushu than in SHR/Izm (i.e., substrain differences) [24]. Because blood pressure levels were the same between the two substrains, factors other than hypertension probably account for different lesion size. The ultraviolet laser-induced reperfusion method, indicated as YAG reperfusion in Figure 1, was also achieved by Watson et al., [26] which was applied to SHR/Kyushu [23] and later to SHR/Izm [27].

\section{Intraluminal suture occlusion}

The intraluminal suture model, developed by Koizumi et al. [28] and Longa et al. [29], is undoubtedly the most frequently used focal ischemia model in rats and mice [30]. To our knowledge, Kawamura et al. first adopted SHR for intraluminal suture model followed by a number of experiments with this method [31]. Alkayed et al. investigated gender-linked brain injury in experimental stroke produced by intraluminal suture occlusion in SHRSP maintained in Johns Hopkins University from a stock obtained from the NIH [32]. Some of the major advantages of the intraluminal suture model are that it is easy to perform, minimally invasive, and most importantly does not require craniectomy. However, it appears that SHR can be replaced with normotensive rats in many experiments with the use of the intraluminal suture occlusion method except for the studies on antihypertensive agents.

Although reperfusion of ischemic brain tissue is critical for restoring normal function, it can paradoxically result in secondary damage or reperfusion injury. Several lines of evidence suggest that post-ischemic oxidative stress and inflammation contribute to brain injury [33]. As reperfusion injury is the condition of ischemia aggravated by the occurrence of reperfusion more so than in the case where reperfusion does not occur (i.e., permanent 
occlusion), in order to see reperfusion injury it is necessary to make a comparison with permanent occlusion. From a critical point of view, the intraluminal suture model is a top of the internal carotid artery occlusion model rather than a MCAO model [34]. Consequently, this model has a wide ischemic zone, and because the mortality rate is high in the case of permanent occlusion, the permanent occlusion group and the reperfusion group that shared the same time lapse cannot be compared.

The intraluminal suture model in SHR had a high incidence of parenchymal hematomas, and therefore is appropriate for the evaluation of reperfusion-associated hemorrhagic transformation [35]. Hemorrhagic transformation is one of the ultimate forms of reperfusion injury. SHR subjected to $3 \mathrm{~h}$ of transient suture MCAO had significant vascular injury or hemorrhagic infarction compared to normotensive rats [36]. Yamashita et al. demonstrated that tPA administered just before the reperfusion of $4.5 \mathrm{~h}$ suture MCAO induced dissociation of the neurovascular unit (i.e., the detachment of astrocyte endfeet from the basement membrane), which was prevented by a free radical scavenger, edaravone [37].

\section{Blood clot embolism}

Although fibrinolytic therapy with tissue plasminogen activator (tPA) is effective in the treatment of acute stroke, there is an elevated risk of brain hemorrhage [38,39]. Hypertension is one of the factors related to the increased incidence of hemorrhagic complications after tPA treatment [40]. A novel model of tPA-induced hemorrhage was examined in an embolic focal ischemia model with homologous blood clots in SHR [41]. Blood pressure was considered to be a critical correlate, because tPA-induced extensive hemorrhagic transformation was observed in SHR but not in normotensive WKY. Since reduction of blood pressure with hydralazine, administered in the drinking water for 1 week before MCAO, significantly reduced the incidence of hemorrhagic transformation, elevated blood pressure during tPA-induced reperfusion is considered to contribute to the pathogenesis of hemorrhage [42]. Therefore, SHR are relevant as a model of embolic stroke as they demonstrate hemorrhagic transformation after tPA therapy.

In the SHR clot embolism model, one study showed a reduction of secondary hemorrhage after thrombolysis combined with normobaric and hyperbaric oxygen therapy [43], while normobaric oxygen did not interfere with the beneficial action of tPA in another study [44].

\section{Penumbra and the SHR stroke models}

Penumbra, analogous to the half-shaded zone around a solar eclipse, is the zone of salvageable tissue around the ischemic core (Figure 2). The classic concept of ischemic penumbra is defined as the condition of an ischemic

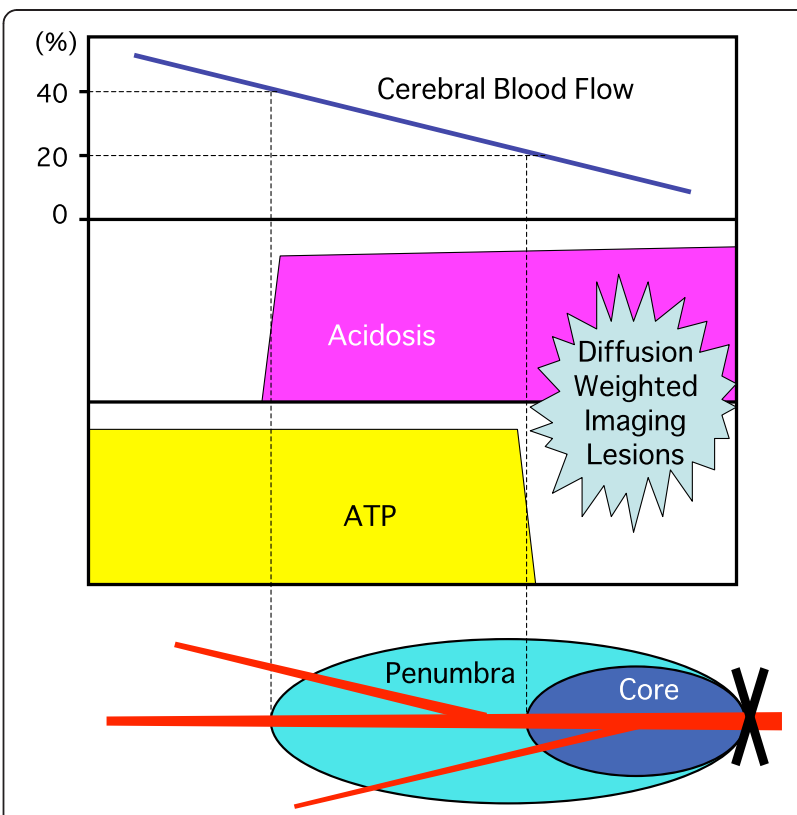

Figure 2 Schematic representation of the ischemic penumbra. Glucose utilization (anaerobic glycolysis) transiently increases at a CBF value below about $40 \%$ of normal, which corresponds to the beginning of acidosis with lactate accumulation. At a CBF value below about $20 \%$ of normal, a breakdown of energy state begins with reduced adenosine triphosphate (ATP), resulting in the condition of ischemic core. The penumbra is defined as an area in which metabolism is impaired due to reduced CBF, but the cellular polarization is still maintained without diffusion- weighted imaging lesions on magnetic resonance imaging. Therefore, an acidosis/ diffusion- weighted imaging mismatch represents penumbra.

brain with CBF between the upper threshold of electrical silence and the lower threshold of energy and ion pump failure $[18,45]$. Regions having $\mathrm{CBF}$ in the ischemic core range (CBF $0 \%$ to $20 \%$ of control values) had a $96 \%$ probability of undergoing infarction, and zones of higher CBF ( $>40 \%$ of control) were largely spared from infarction [46]. One of the most sensitive biochemical markers of cerebral ischemia is the inhibition of protein synthesis, which occurs above the upper threshold of classical penumbra. Under experimental conditions, the most reliable way to localize the ischemic core is the loss of adenosine triphosphate (ATP), and the biochemical marker of the core plus penumbra is tissue acidosis [47]. Therefore, $\mathrm{pH}$-weighted magnetic resonance imaging and diffusion-weighted imaging mismatch can provide a more comprehensive zone of penumbra [48]. In the clinical routine, however, perfusion-weighted imaging/ diffusion-weighted imaging mismatch is used as a surrogate marker for the penumbra: the early diffusionweighted imaging lesion might define the ischemic core, while the adjacent critically hypoperfused tissue might be identified as penumbra with perfusion-weighted imaging [49]. Even after early reperfusion with tPA, the sustained 
reversal of diffusion abnormality was minimal, which indicates that the infarct core is well represented by the acute diffusion lesion [50]. The linear increase in brain sodium after MCAO, which indicates ischemic core, was demonstrated directly by sodium magnetic resonance imaging, and therefore sodium magnetic resonance imaging could provide a better measure of tissue viability compared to diffusion-weighted imaging [51].

It has not been clear, however, which of the focal ischemia models in SHR is most relevant to the penumbra concept. Proximal MCAO produces two ischemic vascular territories of lenticulostriate arteries and cortical branches of MCA, the former of which are end-arteries and therefore treatment-resistant with little penumbra. The intraluminal suture model is a type of internal carotid artery occlusion model rather than a pure MCAO model [34]. In clinical settings, the arterial obstruction site strongly predicts infarct growth and clinical outcome: internal carotid artery occlusion carried a uniformly poor prognosis with poor response to tPA therapy [52]. Although the infarcts produced by distal MCAO in normotensive rats are small with presumably limited size of penumbra, distal MCAO alone in SHR results in substantial size of infarction. However, genetic hypertension (i.e., SHR) and induced renovascular hypertension resulted in a larger lesion and smaller penumbra compared with normotensive WKY after intraluminal suture occlusion [53]. In the case of combined carotid artery occlusions, concurrent carotid occlusions may excessively suppress the retrograde collateral flow to the penumbra. Taken together, according to the ischemic penumbra concept, the distal MCAO model without carotid artery involvement is the best choice for human stroke (i.e., one major cerebral artery occlusion with collateral perfusion) among SHR stroke models (Figure 2).

In our experience with distal MCAO in SHR, the early restoration of perfusion confers advantages on the ischemic brain at risk. The cortical region, rescued by early reperfusion, was considered to be the penumbral zone [54]. At $6 \mathrm{~h}$ after MCAO, lesion volume (not infarction), determined with 2,3,5-triphenyltetrazolium chloride (TTC), was the same among the groups of permanent, $1 \mathrm{~h}$, and $2 \mathrm{~h} \mathrm{MCAO}$, whereas $2 \mathrm{~h}$ and permanent MCAO produced larger infarction than $1 \mathrm{~h}$ MCAO by approximately 2-fold after $24 \mathrm{~h}$ to $48 \mathrm{~h}$ [27]. Although TTC methods underestimate the infarct volume because of reactive gliosis, macrophage infiltration, and other proliferative responses in the infarction at later time points [55], TTC staining is a convenient procedure and reliable for detection of brain infarction at $24 \mathrm{~h}$ after the onset of ischemia [56]. Hence, the volume of penumbra is considered to be approximately $50 \%$ of final infarct volume in our model. In the ischemic core, $\mathrm{Na}^{+}$increased and $\mathrm{K}^{+}$ decreased progressively $3 \mathrm{~h}$ to $6 \mathrm{~h}$ after onset of ischemia, while these electrolytes were within normal values in the acute phase of brain ischemia in the penumbral zone [57]. In this stroke model, apoptotic internucleosomal cleavage (DNA ladder), which is energy dependent, was observed in the penumbral zone but not in the ischemic core at $6 \mathrm{~h}$ after MCAO, which was preceded by large DNA fragmentation in both penumbra and core at $3 \mathrm{~h}$ after MCAO [58]. Protein synthesis is inhibited at a CBF value clearly above the disturbance of glucose utilization and energy metabolism. Attenuation or recovery of deranged proteomic profile was slow even after a short period of ischemia (i.e., $1 \mathrm{~h} \mathrm{MCAO}$ ). Nevertheless, it was not permanently disturbed in the reperfused-penumbra, while a derangement in proteomic profile appeared to be an irreversible process in the ischemic core [59]. These results also support the view that the distal MCAO model in SHR provides a rational approach to the penumbra concept.

\section{MCAO experiments in SHR: A systematic review}

We performed an online PubMed search based on the terms "spontaneously hypertensive rats AND focal ischemia" with limits "English, and Animal" and analyzed all original articles published between 2001 and 2010. We also hand-searched 2 journals (Stroke and Journal of Cerebral Blood Flow and Metabolism) that had published a large proportion of relevant material. We identified 118 original articles dealing with focal ischemia in SHR and SHRSP (Table 1). Stroke resistant or regular SHR were used in 109 of 118 experiments (92\%). Recently, SHR have mostly been obtained from commercial suppliers such as Charles River (SHR/NCrl), Taconic Farms (SHR/NTac), Harlan (SHR/NHsd), Janvier, and the Disease Model Cooperative Research Association or Japan SLC (SHR/Izm) (80 of 118 experiments [68\%]), and SHR 19 (16\%) were from research institutes. Models of distal MCAO, distal MCAO combined with ipsilateral common carotid artery occlusion, and photothrombotic distal MCAO were all based on the occlusion of distal portion of MCA: distal MCAO is the predominant method (55 of 118 experiments [47\%]) of focal ischemia in SHR. The intraluminal suture model was also frequently used (42 of 118 experiments [36\%]) as expected.

In SHRSP/Izm, blood pressure was higher, and infarct volume after MCAO was larger than SHR/Izm [27]. However, we found the survival rate after MCAO was lower in SHRSP/Izm than SHR/Izm (87\% vs. 99\%) in our recent experience. In most experiments with focal ischemia, regular SHR are enough for the preclinical testing of stroke therapies and elucidating the pathophysiology of cerebral ischemia. Consequently, SHRSP were not frequently used for focal ischemia models (9 of $118[8 \%])$. 
Table 1 Focal ischemia models in spontaneously hypertensive

\begin{tabular}{|c|c|c|c|c|c|c|c|}
\hline Author & Substrains & Age & BW (g) & MABP $(\mathrm{mmHg})$ & Methods of MCAO & $\mathrm{CBF}$ & References \\
\hline Oyama N, et al. & SHR/Charles River Jpan & $10 w k$ & NA & $124 \pm 5$ (SEM) & intraluminal suture & LDF & J Neurosci Res 2010;88:2889-2898 \\
\hline Zhao $X$, et al. & SHR/Harlan & NA & NA & NA & dMCAO+ipsi-CCAO (Brint) & LDF & Stroke 2010;41:363-367 \\
\hline Kranz A, et al. & SHR/Charles River & NA & 250 & NA & pMCAO (Tamura) & & Brain Res 2010;1315:128-136 \\
\hline Yang $Y$, et al. & $S H R^{*}$ & NA & $300-320$ & NA & intraluminal suture & & J Neurochem 2010;112:134-149 \\
\hline Ito $\mathrm{H}$, et al. & SHR/Hos, Japan SLC & $20-24 w k$ & $346 \pm 33$ & $209 \pm 13$ & photothrombotic dMCAO & LDF & J Cereb Blood Flow Metab 2010;30:343-351 \\
\hline Sun L, et al. & SHR/Janvier France & NA & $300-350$ & $120 \pm 11$ & blood clots embolism & & J Cereb Blood Flow Metab 2010;30:1651-1660 \\
\hline Porritt MJ, et al. & SHR/ARC Australia & $16 \mathrm{mo}$ & $438 \pm 11$ & $S B P=200-210$ & intraluminal suture & & J Cereb Blood Flow Metab 2010;30:1520-1526 \\
\hline Yao H, et al. & SHR/Izm, Japan SLC & 5-7 mo & $352 \pm 12$ & $172 \pm 12$ & photothrombotic dMCAO/Re & LDF & Neurochem Res 2009;34:1999-2007 \\
\hline Yao H, et al. & SHR/Izm, Japan SLC & 5-7 mo & NA & 182 (mean) & photothrombotic dMCAO/Re & LDF & J Cereb Blood Flow Metab 2009;29:565-574 \\
\hline McCabe C, et al. & SHRSP/Gla & $12-16 w k$ & $220-390$ & $93 \pm 4$ & intraluminal suture & MRI & Stroke 2009;40:3864-3868 \\
\hline Yan Y-P, et al. & SHR/Charles River USA & NA & $270-320$ & NA & intraluminal suture & LDF & Neurochem Int 2009;55:826-832 \\
\hline Ashioti M, et al. & SHR/Charles River UK & NA & $200-250$ & NA & dMCAO+ipsi-CCAO & & BMC Neurosci 2009;10:82 \\
\hline Ishikawa E, et al. & SHR/Kyushu & $5-8 \mathrm{mo}$ & $360-440$ & $154 \pm 11$ & photothrombotic dMCAO & LDF & J Neurol Sci 2009;285:78-84 \\
\hline Omura-Matsuoka E, et al. & SHR/Charles River Jpan & $10-15 w k$ & $250-350$ & $181 \pm 10$ & intraluminal suture & LDF & Hypertens Res 2009;32:548-553 \\
\hline Cao F, et al. & SHRSP* & $13-15 w k$ & $250-300$ & NA & dMCAO & & Brain Res 2009;1272:52-61 \\
\hline Liu Y-P, et al. & SHR/Charles River USA & NA & 290-310 & $130-140$ & intraluminal suture & LDF & J Cereb Blood Flow Metab 2009;29:780-791 \\
\hline Sasaki T, et al. & SHR/Charles River Jpan & NA & $320 \pm 40$ & NA & dMCAO+ipsi-CCAO (Brint) & LDF & Neurosci Lett 2009;449:61-65 \\
\hline Henning EC, et al. & SHR/Charles River USA & $3-4 \mathrm{mo}$ & $350 \pm 20$ & NA & intraluminal suture & & J Cereb Blood Flow Metab 2009;29:1229-1239 \\
\hline Dharap A, et al. & SHR/Charles River USA & NA & $280-300$ & NA & intraluminal suture & & J Cereb Blood Flow Metab 2009;29:675-687 \\
\hline Ishibashi S, et al. & SHR/Charles River USA & $8 w k$ & $180-220$ & NA & $\mathrm{dMCAO}$ & & J Cereb Blood Flow Metab 2009;29:606-620 \\
\hline Yamashita T, et al. & SHR/Izm, Japan SLC & $13 w k$ & $250-280$ & $117 \pm 29$ & intraluminal suture & LDF & J Cereb Blood Flow Metab 2009;29:715-725 \\
\hline Fujiwara N, et al. & SHR/Charles River USA & NA & $303 \pm 8$ & $183 \pm 6$ & blood clots embolism & LDF & BMC Neurosci 2009;10:79 \\
\hline Murata Y, et al. & SHR/Charles River USA & NA & $283 \pm 8$ & $184 \pm 5$ & blood clots embolism & LDF & Stroke 2008;39:3372-3377 \\
\hline Matsuzaki T, et al. & SHR/Izm, Japan SLC & 9 wk & 240-290 & $126 \pm 17$ & intraluminal suture & LDF & Neurol Res 2008;30:531-535 \\
\hline Kozak W, et al. & SHR/Charles River USA & $12-13 w k$ & $275-300$ & $135 \pm 8$ (telemetry) & intraluminal suture & LDF & J Pharmacol Exp Ther 2008;326:773-782 \\
\hline Henning EC, et al. & SHR/Charles River USA & $3-4 \mathrm{mo}$ & $300-360$ & $S B P=165-185$ & intraluminal suture & & Stroke 2008;39:3405-3410 \\
\hline Park S-W, et al. & SHR/Charles River USA & NA & $250-350$ & NA & intraluminal suture & LDF & J Neurosurg 2007;107:593-599 \\
\hline Korde AS, et al. & SHR/Harlan & NA & $250-350$ & $94 \pm 3$ & dMCAO+ipsi-CCAO (Brint) & LDF & J Neurotrauma 2007;24:895-908 \\
\hline Kurasako T, et al. & SHR/Harlan & NA & $220-300$ & $101 \pm 12$ & dMCAO+ipsi-CCAO (Brint) & & J Cereb Blood Flow Metab 2007;27:1919-1930 \\
\hline Jimenez-Altayo F, et al. & SHR/Janvier Spain & $13-15 w k$ & $341 \pm 4$ (SEM) & $S B P=204 \pm 2(S E M)$ & intraluminal suture & & Am J Physiol Heart Circ Physiol 2007;293:H628-635 \\
\hline Tureyen K, et al. & SHR/Charles River USA & NA & NA & $135 \pm 9$ & intraluminal suture & LDF & J Neurochem 2007;101:41-56 \\
\hline Yao H, et al. & SHRSP/Izm, Japan SLC & $5 \mathrm{mo}$ & $315 \pm 30$ & $241 \pm 22$ & photothrombotic dMCAO & LDF & Physiol Genomics 2007;30:69-73 \\
\hline Ashioti M, et al. & SHR/Charles River UK & NA & $265 \pm 9$ & NA & dMCAO+ipsi-CCAO & & Brain Res 2007;1145:177-189 \\
\hline
\end{tabular}


Table 1 Focal ischemia models in spontaneously hypertensive (Continued)

\begin{tabular}{|c|c|c|c|c|c|c|c|}
\hline Mariucci G, et al. & SHR/Charles River Italy & $12-14 w k$ & $250-330$ & NA & pMCAO (Tamura) & & Neurosci Lett 2007;415:77-80 \\
\hline Adibhatla RM, et al. & SHR/Charles River USA & NA & $250-300$ & NA & intraluminal suture & & Brain Res 2007;1134:199-205 \\
\hline Leker RR, et al. & SHR/Tel Aviv & NA & NA & NA & dMCAO & & Stroke 2007;38:153-161 \\
\hline Kumai Y, et al. & SHR/Kyushu & $5-8 \mathrm{mo}$ & $340-435$ & $164 \pm 2(\mathrm{SEM})$ & photothrombotic dMCAO & LDF & J Cereb Blood Flow Metab 2007;27:1152-1160 \\
\hline Yang $Y$, et al. & $S H R^{*}$ & NA & $300-320$ & NA & intraluminal suture & & J Cereb Blood Flow Metab 2007;27:697-709 \\
\hline Yan Y-P, et al. & SHR/Charles River USA & NA & $270-300$ & NA & intraluminal suture & LDF & J Cereb Blood Flow Metab 2007;27:1213-1224 \\
\hline Takasawa M, et al. & SHR/Charles River UK & NA & 295-305 & NA & dMCAO+ipsi-CCAO & & J Cereb Blood Flow Metab 2007;27:679-689 \\
\hline Akaiwa K, et al. & $S H R^{*}$ & NA & $250-290$ & $144 \pm 5$ & intraluminal suture & LDF & Brain Res 2006;1122:47-55 \\
\hline Yan Y-P, et al. & SHR/Charles River USA & NA & $250-300$ & $130-140$ & intraluminal suture & LDF & Eur J Neurosci 2006;24:45-54 \\
\hline Lammer A, et al. & SHR/Charles River Germany & NA & $250-300$ & NA & pMCAO (Tamura) & & Eur J Neurosci 2006;23:2824-2828 \\
\hline Bowen KK, et al. & SHR/Charles River USA & NA & $280-320$ & NA & intraluminal suture & LDF & Neurochem Int 2006;49:127-135 \\
\hline Komitova M, et al. & SHR/Mollegaard & $6 \mathrm{mo}$ & NA & NA & dMCAO & & Exp Neurol 2006;199:113-121 \\
\hline Zhao L, et al. & SHR/Charles River USA & NA & $250-350$ & $118 \pm 12$ & dMCAO+ipsi-CCAO (Brint) & $\mathrm{AR} / \mathrm{LDF}$ & J Cereb Blood Flow Metab 2006;26:1128-1140 \\
\hline Zhang B, et al. & SHRSP* & $16-18 w k$ & NA & $204 \pm 7$ & dMCAO & & J Cereb Blood Flow Metab 2006;26:708-721 \\
\hline Furuya K, et al. & SHR/Charles River Jpan & NA & $280-320$ & NA & dMCAO+ipsi-CCAO (Brint) & LDF & J Neurosurg 2005;103:715-723 \\
\hline Kamiya T, et al. & SHR/Harlan & NA & $225-325$ & $161 \pm 13$ & dMCAO+ipsi-CCAO (Brint) & $A R$ & Stroke 2005;36:2463-2467 \\
\hline Naylor M, et al. & SHR/Charles River USA & NA & $280-320$ & NA & intraluminal suture & LDF & Neurochem Int 2005;47:565-572 \\
\hline Adibhatla RM, et al. & $\mathrm{SHR}^{*}$ & NA & $250-275$ & NA & intraluminal suture & LDF & Brain Res 2005;1058:193-197 \\
\hline Tsuji K, et al. & $S H R^{*}$ & NA & $260-280$ & NA & intraluminal suture & LDF & Stroke 2005;36:1954-1959 \\
\hline Gunther A, et al. & SHR/Charles River Germany & NA & $250-330$ & NA & pMCAO (Tamura) & & Eur J Neurosci 2005;21:3189-3194 \\
\hline Vemuganti R & SHR/Charles River USA & NA & $280-320$ & NA & intraluminal suture & LDF & Neurochem Int 2005;47:136-142 \\
\hline Komitova M, et al. & SHR/Mollegaard & $6 \mathrm{mo}$ & $300-370$ & NA & dMCAO & & Eur J Neurosci 2005;21:2397-2405 \\
\hline Onoue $S$, et al. & SHRSP* & NA & $280-320$ & NA & dMCAO+ipsi-CCAO (Brint) & & Brain Res Mol Brain Res 2005;134:189-197 \\
\hline Hobohm C, et al. & SHR/Charles River Germany & NA & $250-330$ & NA & pMCAO (Tamura) & & J Neurosci Res 2005;80:539-548 \\
\hline Drummond JC, et al. & SHR/Harlan & $16-20 w k$ & $375-425$ & $131 \pm 9$ & intraluminal suture & & Anesth Analg 2005;100:841-846 \\
\hline Ooboshi H, et al. & SHR/Kyushu & $5-8 \mathrm{mo}$ & $340-430$ & NA & photothrombotic dMCAO & LDF & Circulation 2005;111:913-919 \\
\hline Takada J, et al. & SHR/Kyushu & 7-8 mo & $352-450$ & $153 \pm 10$ & photothrombotic dMCAO & LDF & Gene Ther 2005;12:487-493 \\
\hline Kumai Y, et al. & SHR/Kyushu & 5-10 mo & $320-400$ & $181 \pm 21$ & photothrombotic dMCAO & LDF & J Cereb Blood Flow Metab 2004;24:1359-1368 \\
\hline Carswell HV, et al. & SHRSP/Gla, female & $3-4$ mo & $179 \pm 11$ & $140 \pm 15$ & dMCAO & & J Cereb Blood Flow Metab 2004;24:298-304 \\
\hline Franke $\mathrm{H}$, et al. & SHR/Charles River Germany & NA & $250-330$ & NA & pMCAO (Tamura) & & J Neuropathol Exp Neurol 2004;63:686-699 \\
\hline Zhang B, et al. & SHRSP* & $12-13 w k$ & $250-300$ & NA & dMCAO & & Neuroscience 2004;126:433-440 \\
\hline Dhodda VK, et al. & SHR/Charles River USA & NA & $280-320$ & NA & intraluminal suture & LDF & J Neurochem 2004;89:73-89 \\
\hline Nurmi A, et al. & SHR/M\&B A/S & NA & NA & NA & PMCAO & & Stroke 2004;35:987-991 \\
\hline Vemuganti R, et al. & SHR/Charles River USA & NA & $280-320$ & $124 \pm 11$ & intraluminal suture & LDF & Stroke 2004;35:179-184 \\
\hline
\end{tabular}


Table 1 Focal ischemia models in spontaneously hypertensive (Continued)

\begin{tabular}{|c|c|c|c|c|c|c|c|}
\hline Ren $Y$, et al. & SHR/Harlan & NA & 250-300 & $124 \pm 16$ & dMCAO+ipsi-CCAO (Brint) & & J Cereb Blood Flow Metab 2004;24:42-53 \\
\hline Kumai Y, et al. & SHR/Kyushu, female & 5-7 mo & $195-240$ & $170 \pm 2(S E M)$ & photothrombotic dMCAO & LDF & Exp Neurol 2003;184:904-911 \\
\hline Babu GN, et al. & $S H R^{*}$ & NA & $250-300$ & NA & intraluminal suture & LDF & Neurochem Res 2003;28:1851-1857 \\
\hline Gautier S, et al. & SHR/Elevage Janvier France & NA & $270-320$ & NA & intraluminal suture & & Stroke 2003;34:2975-2979 \\
\hline Asahi M, et al. & SHR/Taconic Farms & NA & NA & NA & blood clots embolism & LDF & J Cereb Blood Flow Metab 2003;23:895-899. \\
\hline Teichner A, et al. & $S H R^{*}$ & $13 w k$ & NA & NA & dMCAO & & Exp Neurol 2003;182:353-360 \\
\hline Sadanaga-Akiyoshi F, et al. & SHR/Kyushu & 5-7 mo & $315-425$ & $178 \pm 11 / 176 \pm 7$ & photothrombotic dMCAO & LDF & Neurochem Res 2003;28:1227-1234 \\
\hline Dahlqvist $P$, et al. & SHR/Mollegaard & $14-15$ wk & NA & NA & dMCAO & & Neuroscience 2003;119:643-652 \\
\hline Pettigrew LC, et al. & SHR/Harlan & NA & $250-300$ & $98 \pm 17$ & dMCAO+ipsi-CCAO (Brint) & & Neurol Res 2003;25:201-207 \\
\hline Yan Y, et al. & SHR/Charles River USA & NA & $270-310$ & $133 \pm 9$ & intraluminal suture & & Brain Res 2003;961:22-31 \\
\hline Yamakawa $\mathrm{H}$, et al. & SHR/Taconic Farms & $12 w k$ & $300-500$ & $S B P=156 \pm 4$ & dMCAO & & J Cereb Blood Flow Metab 2003;23:371-380 \\
\hline Wiessner $C$, et al. & SHR/Iffa Credo & NA & $220-300$ & NA & dMCAO & & J Cereb Blood Flow Metab 2003;23:154-165 \\
\hline Rao VLR, et al. & SHR/Charles River USA & NA & $280-320$ & NA & intraluminal suture & LDF & J Neurochem 2002;83:1072-1086 \\
\hline Aoki T, et al. & $S H R^{*}$ & NA & NA & $177 \pm 6$ & blood clots embolism & LDF & Stroke 2002;33:2711-2717 \\
\hline Leker RR, et al. & SHR/Tel Aviv & $13 w k$ & NA & $127 \pm 11$ & dMCAO & LDF & Exp Neurol 2002;176:355-363 \\
\hline Leker RR, et al. & SHR/Tel Aviv & $13 w k$ & NA & $127 \pm 5$ & dMCAO & LDF & Stroke 2002;33:1085-1092 \\
\hline Adibhatla RM, et al. & $S H R^{*}$ & NA & NA & NA & intraluminal suture & & Brain Res 2002;938:81-86 \\
\hline Sakakibara Y, et al. & SHR/Charles River USA & NA & $280-350$ & $116-128$ & intraluminal suture & & Brain Res 2002;931:68-73 \\
\hline Sumii T, et al. & SHR/Taconic Farms & NA & NA & $185 \pm 6$ (SEM) & blood clots embolism & LDF & Stroke 2002;33:831-836 \\
\hline Jin J, et al. & SHR/Hos, Japan SLC & NA & $130-160$ & NA & PMCAO & AR & Pharmacology 2002;64:119-125 \\
\hline Higuchi T, et al. & SHR/Charles River Jpan & NA & $261 \pm 32$ & $88 \pm 12$ & dMCAO+ipsi-CCAO (Brint) & LDF & J Cereb Blood Flow Metab 2002;22:71-79 \\
\hline Ito T, et al. & SHR/Taconic Farms & $8 w k$ & $190-240$ & $S B P=153 \pm 6(S E M)$ & dMCAO & & Stroke 2002;33:2297-2303 \\
\hline Wallace JA, et al. & $S H R^{*}$ & NA & $280-320$ & NA & intraluminal suture & & J Cereb Blood Flow Metab 2002;22:1303-1310 \\
\hline Komitova M, et al. & SHR/Mollegaard & NA & NA & NA & dMCAO & & J Cereb Blood Flow Metab 2002;22:852-860 \\
\hline Johanson BB, et al. & SHR/Mollegaard & $3 \mathrm{mo}$ & NA & NA & dMCAO & & J Cereb Blood Flow Metab 2002;22:89-96 \\
\hline Dijkhuizen RM, et al. & SHR/Taconic Farms & NA & $300-350$ & NA & blood clots embolism & MRI & Stroke 2002;33:2100-2104 \\
\hline Yao H, et al. & SHR/Kyushu & $5-7 \mathrm{mo}$ & $346-444$ & 196 (mean) & photothrombotic dMCAO/Re & LDF & Neuroreport 2002;13:1005-1008 \\
\hline Kitayama J, et al. & SHR/Kyushu & $5-8 \mathrm{mo}$ & $305-438$ & $161 \pm 5$ (SEM) & photothrombotic dMCAO & LDF & Brain Res 2001;922:223-228 \\
\hline Furuya K, et al. & $S H R^{*}$ & NA & NA & NA & dMCAO+ipsi-CCAO (Brint) & & Stroke 2001;32:2665-2674 \\
\hline Takagi K, et al. & SHR/Funabashi Farm Japan & $15-16 w k$ & $280-340$ & $177 \pm 11$ & pMCAO (Tamura) & LDF & Neurol Res 2001;23:662-668 \\
\hline Cole DJ, et al. & $S H R^{*}$ & $16-20 w k$ & $375-425$ & $126 \pm 9 / 131 \pm 7$ & intraluminal suture & & Can J Anaesth 2001;48:807-814 \\
\hline Yan Y, et al. & SHR/Charles River USA & NA & $270-300$ & $133 \pm 8$ & intraluminal suture & & J Cereb Blood Flow Metab 2001;21:711-721 \\
\hline Rao VLR, et al. & SHR/Charles River USA & NA & $250-300$ & $116 \pm 11$ & intraluminal suture & LDF & J Cereb Blood Flow Metab 2001;21:945-954 \\
\hline Zhao Z, et al. & SHR/Charles River Canada & NA & $200-250$ & $127 \pm 14$ & dMCAO+ipsi-CCAO & LDF & Brain Res 2001;909:46-50 \\
\hline
\end{tabular}


Table 1 Focal ischemia models in spontaneously hypertensive (Continued)

\begin{tabular}{|c|c|c|c|c|c|c|c|}
\hline Marks $L$, et al. & SHRSP/Gla & $3-5 \mathrm{mo}$ & $262 \pm 11($ SEM) & $123 \pm 9$ (SEM) & $\mathrm{dMCAO}$ & & Hypertension 2001;38:116-122 \\
\hline Gregersen $\mathrm{R}$, et al. & SHR/Mollegaard & NA & $300-335$ & NA & PMCAO & & Exp Brain Res 2001;138:384-392 \\
\hline Yao H, et al. & SHR/Kyushu & $5-7 \mathrm{mo}$ & $350-438$ & 187 (mean) & photothrombotic dMCAO & LDF & Brain Res Mol Brain Res 2001;91:112-118 \\
\hline Tejima E, et al. & $S H R^{*}$ & NA & $250-300$ & $122 \pm 3($ SEM) & blood clots embolism & LDF & Stroke 2001;32:1336-1340 \\
\hline Lavie G, et al. & SHR/Tel Aviv & $13 w k$ & NA & $127 \pm 11$ & $\mathrm{dMCAO}$ & & Brain Res 2001;901:195-201 \\
\hline Furuya K, et al. & SHR/Charles River USA & $13-16$ wk & NA & $140 \pm 7$ & pMCAO (Tamura) & & J Cereb Blood Flow Metab 2001;21:226-232 \\
\hline Holtz ML, et al. & SHR/Harlan & NA & $250-350$ & $108 \pm 15$ & dMCAO+ipsi-CCAO (Brint) & & Brain Res 2001;898:49-60 \\
\hline Fredduzzi S, et al. & SHR/Charles River Italy & $12-14$ wk & 250-330 & NA & pMCAO (Tamura) & & Neurosci Lett 2001;302:121-124 \\
\hline Rao VLR, et al. & SHR/Charles River USA & NA & $250-300$ & NA & intraluminal suture & LDF & J Neurosci 2001;21:1876-1883 \\
\hline Negrin $C D$, et al. & SHRSP/Gla & $13 w k$ & NA & $\mathrm{SBP}=186 \pm 6(\mathrm{SEM})$ & dMCAO & & Hypertension 2001;37:391-397 \\
\hline Babu GN, et al. & $\mathrm{SHR}^{*}$ & NA & $250-300$ & NA & intraluminal suture & LDF & Neurosci Lett 2001;300:17-20 \\
\hline Legos JJ, et al. & SHR/Taconic Farms & NA & $300-350$ & NA & $\mathrm{dMCAO}$ & & Brain Res 2001;892:70-77 \\
\hline Slivka AP, et al. & $S H R^{*}$ & NA & $240-400$ & NA & dMCAO+ipsi-CCAO (Brint) & & Exp Neurol 2001;167:166-172 \\
\hline Barone FC, et al. & SHR/Taconic Farms & NA & 290-340 & $141 \pm 3($ SEM) & dMCAO & & J Pharmacol Exp Ther 2001;296:312-321 \\
\hline Relton JK, et al. & SHR/Taconic Farms & NA & 250-350 & $168 \pm 15($ SEM) & intraluminal suture & & Stroke 2001;32:199-205 \\
\hline Ooboshi H, et al. & SHR/Kyushu & $5-7 \mathrm{mo}$ & $340-430$ & $167 \pm 6$ (SEM) & photothrombotic dMCAO & LDF & Stroke 2001;32:1043-1047 \\
\hline Dijkhuizen RM, et al. & SHR/Taconic Farms & NA & $300-350$ & NA & blood clots embolism & LDF / MRI & J Cereb Blood Flow Metab 2001;21:964-971 \\
\hline
\end{tabular}

*substrain unknown; NA, not available. Values are mean \pm S.D. BW, body weight; MABP, mean arterial blood pressure;

MCAO, middle cerebral artery occlusion; d, distal; p, proximal; ipsi, ispsilateral; Re, reperfusion;

$\mathrm{CBF}$, cerebral blood flow; LDF, laser Doppler flowmetry; $\mathrm{AR}$, autoradiography; MIR, magnetic resonance imaging 
Resting blood pressure values were available in 62 (53\%) articles, while we could not find a description of blood pressure in 56 (47\%) articles. The information on blood pressure of SHR is apparently lacking in a substantial amount of literature. Of 35 experiments with standard inhalation anesthesia, rats were intubated, and the anesthesia was maintained with low concentrations of halothane or isoflurane in 13 experiments, while high concentrations of anesthesia were used in 22 experiments often with a facemask in the spontaneously breathing animal. Resting MABP levels were significantly lower (median 132, interquartile range [IQR] 122-140) in those with high concentrations of anesthesia than those (median 177, IQR 164-182) with low concentrations of anesthesia (Mann-Whitney u-test, $\mathrm{p}=0.000$ ) (Figure 3). There is general agreement that hypotension during acute stroke is detrimental to perfusion of ischemic brain and tissue outcome [60]. CBF autoregulation was lost when CBF fell below 30\% of normal tissue [61]. Arterial blood pressure had a greater influence on CBF in moderately ischemic brain tissue, suggesting potential benefits from raising and possible harm from lowering intraischemic blood pressure. Zhu and Auer (1995) studied the effects of halothane-induced hypotension on histological outcome after transient intraluminal suture occlusion in Wistar rats [62]. Intraischemic hypotension significantly increased infarct size, and the penumbra, defined here as the zone with selective neuronal necrosis, was lost at a blood pressure of $40 \mathrm{mmHg}$. In line with these observations, mechanical ventilation was required to obtain controlled experimental conditions in focal brain ischemia: spontaneously breathing animals, anesthetized with chloral hydrate i.p. or with halothane via facemask, exhibited respiratory acidosis and decreased blood pressure, resulting in high mortality and a significantly increased infarct volume [63].

In SHR, blood pressure levels are classified into 3 time points as follows: (1) the prehypertensive period (4 weeks), (2) the period of rapidly rising blood pressure (8 and 12 weeks), and (3) sustained hypertension (16-18 weeks) [64]. Blood pressure of SHR rises between 1 and 5 months, and blood pressure as well as body weight remain constant thereafter [65]. Survival rate of male SHR/Kyushu declines sharply after 15 months of age. Infarct volume in the young SHRSP (10-12 weeks) was substantially smaller than that in adult SHRSP (5 months) [66]. On the basis of these observations, SHR should be used at 5 to 7 months and SHRSP at 5 months for adult models of focal ischemia. Age as well as body weight should be described in the manuscript to better understand the effects of age on ischemic insults in SHR. We analyzed a possible chronological variability of physiological variables in adult male SHR/Izm (5-7 months old, $\mathrm{n}=195)$ used between 2005 and 2010 for our stroke research, and found that resting MABP in 2009-2010 (163 \pm 9 [S.D.] $\mathrm{mmHg}$ ) was significantly lower than those in 2005-2006 and 2007-2008 (175 $\pm 10 \mathrm{mmHg}$ and $173 \pm 9 \mathrm{mmHg}$, respectively) (ANOVA and Scheffe test, $p=0.000$ ), while the mean values of body weight were fairly constant (unpublished observation). This observation suggests that MABP could fluctuate even in the closed colony of SHR/Izm under strict monitoring of blood pressure. Physiological parameters of SHR - notably blood pressure, arterial gases, and brain as well as systemic temperature - should

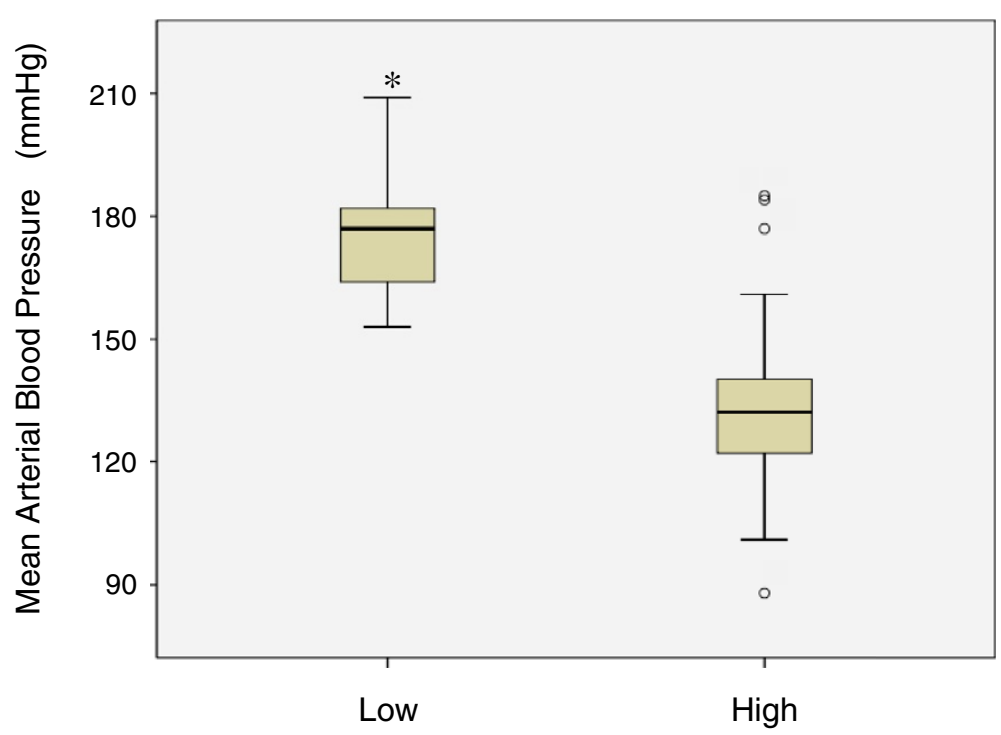

Figure 3 Box and whisker plot of resting mean arterial blood pressure in experiments with low and high concentrations of anesthesia. The box shows median value and 25 th and 75 th percentiles. ${ }^{*} P=0.000$ for difference in mean arterial blood pressure between the groups (Mann-Whitney u-test). 
be closely monitored and regulated in each experiment. The monitoring of brain temperature, reflected by temporalis muscle (or skull) temperature, would be recommended even in closed skull models such as the intraluminal thread model, because studies that used only rectal temperature measurements may be confounded by unknown variations in brain temperature [67].

Sex differences influence both stroke mechanism and response to therapy $[24,68,69]$. However, female SHR were only rarely used ( 2 of 118 ) (Table 1 ).

Among the 118 experiments mentioned above, 58 studies tested the efficacy of neuroprotective strategy (Table 2). We assessed the quality of these studies according to published criteria by Macleod et al. [70] with slight modification. These criteria were: (1) peerreviewed publication, (2) compliance with animal welfare regulations, (3) use of anesthetic without significant intrinsic neuroprotective activity, (4) statement of control of physiological variables, (5) statement of control of brain/head temperature, (6) random allocation to treatment or control, (7) blinded induction of ischemia, (8) blinded assessment of outcome, (9) sample size calculation, and (10) statement of potential conflict of interests. Since statements on conflict of interest have only recently appeared in the pre-clinical stroke literature (2008-2010), this item was not included in the total score, and each study was given a quality score out of a possible total of 9 points. The median quality score was 5.0 (IQR of 4.0-6.0), which was apparently higher than the 3.5 reported by Macleod et al. [70] (experiments published between 1998 and 2003) even considering the minor differences in criteria between the studies, suggesting improved quality of experiments in recent years after the Stroke Therapy Academic Industry Roundtable (STAIR) recommendations [71] and a series of metaanalyses of studies of experimental stroke [70,72,73]. The STAIR-defined criteria for the development and improved clinical testing of neuroprotective drugs are: adequate dose-response data, definition of the time window, blinded and physiologically controlled reproducible studies, hisotological and functional outcomes assessed acutely and long-term, and testing in both permanent and transient MCAO models. High socres $(\geqq 6)$ were largely due to 3 criteria: use of anesthetic without significant intrinsic neuroprotective activity, statement of control of physiological variables, and statement of control of brain/head temperature. A power or sample size calculation was given only in 4 of 58 studies. "Blinded" induction of ischemia or assessment of outcome was described in 8 studies, 7 of which scored high points $(\geqq 6)$. After complete randomization, blinded assessment should be followed for good laboratory practice. Quality of studies with lower scores $(\leqq 4)(21$ of 58 [36\%]) were considered to be inadequate by today's standards. In addition to the criteria mentioned above, we propose that mortality rate after MCAO surgery should be described.

Despite the progress in experimental stroke models, however, the recent angiotensin-receptor blocker candesartan for treatment of acute stroke trial (SCAST) failed to show any beneficial effects in elderly patients with acute stroke and raised blood pressure [74], whereas partial lowering of the blood pressure during reperfusion was beneficial in young SHR [75]. Age may be a factor that accounted for the discrepancy between the preclinical animal experiments and the clinical trial in aged patients. Furthermore, besides the inevitable differences between the animal experiments and human stroke, the most critical point of the disparity in the SCAST scenario was that the animal model mimicked the situation of large vessel occlusion, while a substantial portion of the patients in the SCAST study had the cerebral small vessel disease: $29 \%$ had lacunar syndrome and 14\% hemorrhagic stroke. It is unlikely that a neuroprotective drug acts on lacunar stroke or brain hemorrhage the same as it does on embolic stroke. The mechanistic aspects of the therapeutic strategy should not be left out of accounts of either animal or human studies, since different mechanisms of protection between experimental and clinical settings may lead to failure in translational research.

\section{Cerebral blood flow}

Among the 118 articles (2001-2010) on Table 1, CBF was determined in 60 experiments: 56 with laserDoppler flowmetry, 3 with autoradiography, and 3 with magnetic resonance imaging. One study with autoradiography also adopted 2-dimensional laser-Doppler perfusion imaging (Moor Instruments, Inc.), and in another study both magnetic resonance imaging and laserDoppler flowmetry were used. One of the major advantages of the magnetic resonance imaging method was that this noninvasive modality provided additional information such as diffusion-weighed imaging $[76,77]$ and hemorrhagic transformation [77] in addition to perfusion imaging. The power of $\mathrm{CBF}$ autoradiography lies in its ability to quantitate the local CBF in small discrete brain regions, although flow can be assessed only once and sacrifice of the animal is required. With regard to focal ischemia models, the golden standard of CBF measurement is the quantitative 3-dimensional autoradiography [46]. Laser-Doppler flowmetry is an easy method to monitor a relative measure of blood perfusion, and is commonly used in small animals. Practical considerations in this method include confounding effects such as superfluous light, heterogeneous distribution of superficial blood vessels, and movement artifacts. In the studies of focal ischemia, one-point CBF measurement with laser-Doppler flowmetry may show no 
Table 2 Quality of neuroprotective studies

\begin{tabular}{|c|c|c|c|c|c|c|c|c|c|c|c|c|}
\hline Author & $\begin{array}{r}\text { Year } \\
p\end{array}$ & $\begin{array}{c}(1) \\
\text { peer } \\
\text { reviewed } \\
\text { publication }\end{array}$ & $\begin{array}{c}(2) \\
\text { copmliance } \\
\text { with } \\
\text { animal } \\
\text { welfare }\end{array}$ & $\begin{array}{c}\text { (3) } \\
\text { use of } \\
\text { anesthetics } \\
\text { without } \\
\text { protection }\end{array}$ & $\begin{array}{c}\text { (4) } \\
\text { physiologica } \\
\text { variables } \\
\end{array}$ & $\begin{array}{l}(5) \\
\text { al brain/head } \\
\text { temperaturea }\end{array}$ & $\begin{array}{l}\text { (6) } \\
\text { random } \\
\text { allocation } \\
\text { to Tx or } \\
\text { control }\end{array}$ & $\begin{array}{c}\text { (7) } \\
\text { blinded } \\
\text { induction } \\
\text { of ischemia }\end{array}$ & $\begin{array}{c}\text { (8) } \\
\text { blinded } \\
\text { assessment } \\
\text { aof outcomeca }\end{array}$ & $\begin{array}{c}\text { (9) } \\
\text { sample } \\
\text { size } \\
\text { alculation }\end{array}$ & $\begin{array}{c}\text { (10) } \\
\text { statement } \\
\text { of COI }\end{array}$ & $\begin{array}{c}\text { score } \\
\text { it SUM } \\
\text { of } \\
1 \sim 9\end{array}$ \\
\hline $\begin{array}{l}\text { Fujiwara N, } \\
\text { et al. }\end{array}$ & 2009 & 1 & 1 & 1 & 1 & 0 & 1 & 1 & 0 & 1 & 0 & 7 \\
\hline $\begin{array}{l}\text { Drummond JC, } \\
\text { et al. }\end{array}$ & 2005 & 1 & 1 & 1 & 1 & 1 & 1 & 0 & 1 & 0 & 0 & 7 \\
\hline $\begin{array}{l}\text { Yan Y, } \\
\text { et al. }\end{array}$ & 2001 & 1 & 1 & 1 & 1 & 1 & 1 & 0 & 1 & 0 & 0 & 7 \\
\hline $\begin{array}{l}\text { Ito } \mathrm{H}_{\text {, }} \\
\text { et al. }\end{array}$ & 2010 & 1 & 1 & 1 & 1 & 1 & 1 & 0 & 0 & 0 & 0 & 6 \\
\hline $\begin{array}{l}\text { Ishikawa E, } \\
\text { et al. }\end{array}$ & 2009 & 1 & 1 & 1 & 1 & 1 & 1 & 0 & 0 & 0 & 0 & 6 \\
\hline $\begin{array}{l}\text { Yamashita T, } \\
\text { et al. }\end{array}$ & 2009 & 1 & 1 & 1 & 1 & 1 & 1 & 0 & 0 & 0 & 0 & 6 \\
\hline $\begin{array}{l}\text { Kumai Y, } \\
\text { et al. }\end{array}$ & 2007 & 1 & 1 & 1 & 1 & 1 & 1 & 0 & 0 & 0 & 0 & 6 \\
\hline $\begin{array}{l}\text { Zhang B, } \\
\text { et al. }\end{array}$ & 2006 & 1 & 1 & 1 & 0 & 1 & 1 & 0 & 1 & 0 & 0 & 6 \\
\hline $\begin{array}{l}\text { Kamiya T, } \\
\text { et al. }\end{array}$ & 2005 & 1 & 1 & 1 & 1 & 1 & 1 & 0 & 0 & 0 & 0 & 6 \\
\hline $\begin{array}{l}\text { Ooboshi H, } \\
\text { et al. }\end{array}$ & 2005 & 1 & 1 & 1 & 1 & 1 & 1 & 0 & 0 & 0 & 0 & 6 \\
\hline $\begin{array}{l}\text { Takada J, } \\
\text { et al. }\end{array}$ & 2005 & 1 & 1 & 1 & 1 & 1 & 1 & 0 & 0 & 0 & 0 & 6 \\
\hline $\begin{array}{l}\text { Kumai Y, } \\
\text { et al. }\end{array}$ & 2004 & 1 & 1 & 1 & 1 & 1 & 1 & 0 & 0 & 0 & 0 & 6 \\
\hline $\begin{array}{l}\text { Vemuganti } \\
\text { et al. }\end{array}$ & $R, 2004$ & 1 & 1 & 1 & 1 & 1 & 1 & 0 & 0 & 0 & 0 & 6 \\
\hline Ren $Y$, et al. & 2004 & 1 & 1 & 1 & 1 & 1 & 1 & 0 & 0 & 0 & 0 & 6 \\
\hline $\begin{array}{l}\text { Sadanaga- } \\
\text { Akiyoshi F, et al. }\end{array}$ & 2003 & 1 & 1 & 1 & 1 & 1 & 1 & 0 & 0 & 0 & 0 & 6 \\
\hline Yan Y, et al. & 2003 & 1 & 1 & 1 & 1 & 1 & 1 & 0 & 0 & 0 & 0 & 6 \\
\hline $\begin{array}{l}\text { Sakakibara } \\
\text { et al. }\end{array}$ & Y,2002 & 1 & 1 & 1 & 1 & 0 & 1 & 0 & 1 & 0 & 0 & 6 \\
\hline $\begin{array}{l}\text { Kitayama J, } \\
\text { et al. }\end{array}$ & 2001 & 1 & 1 & 1 & 1 & 1 & 1 & 0 & 0 & 0 & 0 & 6 \\
\hline $\begin{array}{l}\text { Furuya K, } \\
\text { et al. }\end{array}$ & 2001 & 1 & 0 & 1 & 1 & 1 & 1 & 0 & 1 & 0 & 0 & 6 \\
\hline $\begin{array}{l}\text { Rao VLR, } \\
\text { et al. }\end{array}$ & 2001 & 1 & 1 & 1 & 1 & 1 & 1 & 0 & 0 & 0 & 0 & 6 \\
\hline $\begin{array}{l}\text { Furuya K, } \\
\text { et al. }\end{array}$ & 2001 & 1 & 1 & 1 & 1 & 1 & 1 & 0 & 0 & 0 & 0 & 6 \\
\hline $\begin{array}{l}\text { Rao VLR, } \\
\text { et al. }\end{array}$ & 2001 & 1 & 1 & 1 & 1 & 1 & 1 & 0 & 0 & 0 & 0 & 6 \\
\hline $\begin{array}{l}\text { Slivka AP, } \\
\text { et al. }\end{array}$ & 2001 & 1 & 1 & 1 & 1 & 0 & 1 & 0 & 1 & 0 & 0 & 6 \\
\hline $\begin{array}{l}\text { Oyama N, } \\
\text { et al. }\end{array}$ & 2010 & 1 & 1 & 1 & 1 & 0 & 1 & 0 & 0 & 0 & 0 & 5 \\
\hline $\begin{array}{l}\text { Sun } L \text {, } \\
\text { et al. }\end{array}$ & 2010 & 1 & 1 & 1 & 1 & 0 & 1 & 0 & 0 & 0 & 1 & 5 \\
\hline $\begin{array}{l}\text { Ashioti M, } \\
\text { et al. }\end{array}$ & 2009 & 1 & 1 & 1 & 0 & 0 & 1 & 0 & 0 & 1 & 0 & 5 \\
\hline
\end{tabular}


Table 2 Quality of neuroprotective studies (Continued)

\begin{tabular}{llllll}
\hline Liu Y-P, & 2009 & 1 & 1 & 1
\end{tabular}

et al.

et al.

Matsuzaki T, 2008

et al.

Korde AS,

et al.

et al.

Adibhatla $\quad \mathrm{RM}, 2005$

et al.

Carswell HV, 2004

et al.

Asahi M

et al.

Pettigrew LC,2003

et al.

Takagi K, et al. 200

Zhao Z, et al. 2001

Zhao X, et al. 2010

Porritt MJ, et al. 2010

Omura-Matsuoka 2009

E, et al.

Kozak W, et al. $2008 \quad 1 \quad 1 \quad 1$,

Zhang B, et al. $2004 \quad 1$

Nurmi A, et al. $2004 \quad 1$

Wiessner C, 2003

et al.

Leker RR, et al. 2002

Jin J, et al. 2002

Lavie $\mathrm{G}$, et al. 2001

Relton JK, et al. 200

Lammer A, 2006

et al.

Gunther A 2005

et al.

Teichner A, $2003 \quad 1$

et al.

Yamakawa $\quad \mathrm{H}, 2003$

et al.

Ito T, et al. $\quad \begin{array}{llll}2002 & 1 & 1 & 0\end{array}$

Fredduzzi S, 2001

et al.

Barone FC, 2001

et al.

Kranz A, et al. $2010 \quad 1 \quad 0 \quad 0$

Leker RR, et al. $2002 \quad 1 \quad 000$

Legos JJ, et al. 2001

0

0


significant (a false negative) difference in CBF after MCAO between the groups. To overcome this issue, we determined $\mathrm{CBF}$ with the laser-Doppler flowmetry "scanning" method as previously described [66]: a laserDoppler flowmetry probe was laterally scanned, and CBF of the distal MCA territory was measured at five points ( $2 \mathrm{~mm}$ posterior and 2.0, 2.5, 3.0, 3.5, and $4.0 \mathrm{~mm}$ lateral to the bregma), and $5 \mathrm{CBF}$ values in one rat were transformed to an area under curve according to the trapezoidal rule. This "scanning" method worked well, but it is time-consuming and requires much effort. Twodimensional cortical CBF mapping is possible with laserDoppler perfusion imaging, which is a sophisticated and robust image registration $[78,79]$. This system, however, requires an expensive set-up.

\section{Beyond substrains}

To overcome the genetic heterogeneity in substrains of SHR and SHRSP, several approaches are currently in progress. In order to establish a system to facilitate the systematic collection of rat strains and genetic characterization, the National BioResource Project-Rat (NBRP-Rat) was launched in 2002 [80]. By the end of 2008, more than 500 rat strains, including substrains of SHR and SHRSP, had been deposited in the NBRP-Rat. With this database, researchers can select a rat strain (e.g., SHR/Izm) to instantly compare its genetic background against all rat strains typed at NBRP-Rat [81]. Such a phenotypic 'Strain Ranking' allows visual data scoring, which provides an opportunity to easily and simultaneously compare phenotypic values for multiple rat strains. This database could in theory be applied to find genetic differences between the substrains with a different phenotype. Needless to say, this approach will not immediately resolve the major heterogeneity between strains, but a huge rat repository is a reasonable attempt.

Another approach is the congenic strategy based on the genome-wide linkage or quantitative trait loci (QTL) analysis to examine the effects of polygenic trait on the stroke phenotype. Jeffs et al. identified 3 major QTL on chromosomes 1, 4, and 5 related to stroke sensitivity or large infarct size in the SHRSP after MCAO by performing a genome scan in an F2 cross of SHRSPGla and WKYGla [82]. Recently, a novel gene-targeting technology utilizing zinc-finger nucleuses, which does not rely on using species-specific embryonic stem cell lines, has been proved to work successfully even in rats to generate a knockout of specific DNA regions [83]. However, this method is not applicable to the search for new genetic traits in SHR or SHRSP, because the genes or specific DNA regions for essential hypertension or stroke have not been identified in either animals or human beings. Almost all common diseases including stroke are based on polygenes. Stroke falls within the category of complex traits, arising from numerous gene-gene and gene-environment interactions $[84,85]$. To overcome this complexity, the role of identified QTL on stroke susceptibility is currently studied through the production of congenic lines: one or several QTL can be replaced with those of the control strain to examine functional evidence for the role of genes distinct between the two strains. For example, a blood pressure QTL on rat chromosome 1 was introgressed from WKY/Izm to SHRSP/Izm by repeated backcrossing to cancel out the heterogeneity in genetic background between the strains. This congenic removal of the blood pressure QTL increased collateral CBF after MCAO, and despite a small decrease in blood pressure, demonstrated a substantial reduction in infarct volume after MCAO compared with SHRSP/Izm, showing a beneficial effect beyond blood pressure [66]. Congenic substrains are used to narrow down the implicated congenic segment to aid the identification of positional candidate genes [86]. As Aitman et al. showed, microarray combined with congenic strategy may lead to the identification of a causative gene once congenic strains containing smaller QTL have been produced [87]. The congenic strategy would be particularly useful to investigate the substrain differences in SHR and SHRSP or stroke proneness in the future.

\section{Concluding comments}

We have reviewed the early development of various focal ischemia models in substrains of SHR, and summarized recent reports on this topic. Although SHR, which have comorbidity (i.e., spontaneous hypertension), are suitable for stroke research, only $11 \%$ of animal experiments on neuroprotection involved testing in hypertensive rats [30]. Distal MCAO without carotid occlusions in SHR is best suited for the penumbra concept. To translate the results in animal models into human stroke, common mechanisms underlying the ischemic injury or protection by therapy need to be elucidated both in the animal model and human stroke. One of the confounding factors is that genetic heterogeneity among substrains of SHR and SHRSP may make the situation complicated. Therefore, we attempted to provide an overview of focal ischemia models in diverse substrains of SHR. Spin-off findings from recent studies were that age, body weight, and even blood pressure of SHR were not indicated in a number of articles. We would like to emphasize the importance of these basic physiological parameters in light of the different phenotypes among substrains of SHR.

\section{Competing interests}

The authors declare that they have no competing interest.

\section{Authors' contributions}

HY conducted the literature search and wrote manuscript. TN is responsible for critically revising for intellectual content. Both authors read and approved the final manuscript. 


\section{Acknowledgements}

We thank Toshiyuki Sasaguri, M.D. for helpful discussion, and Katherine Kawaharada for editing the manuscript.

\section{Author details}

'Laboratory for Neurochemistry, Center for Emotional and Behavioral Disorders, National Hospital Organization Hizen Psychiatric Center, Mitsu 160, Yoshinogari, Kanzaki, Saga, 842-0192, Japan. ${ }^{2}$ Department of Functional Pathology, Shimane University School of Medicine, Izumo, Japan.

Received: 15 March 2012 Accepted: 2 July 2012

Published: 6 July 2012

\section{References}

1. Okamoto K, Aoki K: Development of a strain of spontaneously hypertensive rats. Jpn Circ J 1963, 27:282-293.

2. Okamoto $K$, Yamori Y, Nagaoka A: Establishment of the stroke-prone spontaneously hypertensive rat. Circ Res 1974, 34/35:1143-1153.

3. Jacewicz M, Tanabe J, Pulsinelli WA: The CBF threshold and dynamics for focal cerebral infarction in spontaneously hypertensive rats. $J$ Cereb Blood Flow Metab 1992, 12:359-370.

4. Kaplan B, Brint S, Tanabe J, Jacewicz M, Wang XJ, Pulsinelli W: Temporal thresholds for neocortical infarction in rats subjected to reversible focal cerebral ischemia. Stroke 1991, 22:1032-1039.

5. Nabika T, Nara Y, Ikeda K, Endo J, Yamori Y: Genetic heterogeneity of the spontaneously hypertensive rat. Hypertension 1991, 18:12-16.

6. St Lezin E, Simonet L, Pravenec M, Kurtz TW: Hypertensive strains and normotensive 'control' strains. How closely are they related? Hypertension 1992, 19:419-424.

7. Johnson ML, Ely DL, Turner ME: Genetic divergence between the Wistar-Kyoto rat and the spontaneously hypertensive rat. Hypertension 1992, 19:425-427.

8. Yamori Y, Horie R, Handa H, Sato M, Fukase M: Pathogenetic similarity of strokes in stroke-prone spontaneously hypertensive rats and humans. Stroke 1976, 7:46-53.

9. Bailey EL, Smith C, Sudlow CL, Wardlaw JM: Is the spontaneously hypertensive stroke prone rat a pertinent model of sub cortical ischemic stroke? A systematic review. Int I Stroke. 2011, 6:434-444.

10. Hainsworth AH, Markus HS: Do in vivo experimental models reflect human cerebral small vessel disease? A systematic review. I Cereb Blood Flow Metab 2008, 28:1877-1891.

11. Nabika T, Cui Z, Masuda J: The stroke-prone spontaneously hypertensive rat: how good is it as a model for cerebrovascular diseases? Cell Mol Neurobiol 2004, 24:639-646.

12. Rubattu S, Hubner N, Ganten U, Evangelista A, Stanzione R, Di Angelantonio E, Plehm R, Langanki R, Gianazza E, Sironi L, D'Amati G, Volpe M: Reciprocal congenic lines for a major stroke QTL on rat chromosome 1. Physiol Genomics 2006, 27:108-113.

13. Tamura A, Graham DI, McCulloch J, Teasdale GM: Focal cerebral ischaemia in the rat: 1. Description of technique and early neuropathological consequences following middle cerebral artery occlusion. J Cereb Blood Flow Metab 1981, 1:53-60.

14. Coyle P, Jokelainen PT: Differential outcome to middle cerebral artery occlusion in spontaneously hypertensive stroke-prone rats (SHRSP) and Wistar Kyoto (WKY) rats. Stroke 1983, 14:605-611.

15. Duverger D, MacKenzie ET: The quantification of cerebral infarction following focal ischemia in the rat: influence of strain, arterial pressure, blood glucose concentration, and age. J Cereb Blood Flow Metab 1988, 8:449-461.

16. Brint $\mathrm{S}$, Jacewicz M, Kiessling M, Tanabe J, Pulsinelli W: Focal brain ischemia in the rat: methods for reproducible neocortical infarction using tandem occlusion of the distal middle cerebral and ipsilateral common carotid arteries. J Cereb Blood Flow Metab 1988, 8:474-485.

17. Yao H, Sugimori H, Fukuda K, Takada J, Ooboshi H, Kitazono T, Ibayashi S, lida M: Photothrombotic middle cerebral artery occlusion and reperfusion laser system in spontaneously hypertensive rats. Stroke 2003, $34: 2716-2721$

18. Astrup J, Siesjö BK, Symon L: Thresholds in cerebral ischemia - the ischemic penumbra. Stroke 1981, 12:723-725.

19. Furspan PB, Rinaldi GJ, Hoffman K, Bohr DF: Dietary calcium and cell membrane abnormality in genetic hypertension. Hypertension 1989, 13:727-730.
20. Fujii K, Weno BL, Baumbach GL, Heistad DD: Effect of antihypertensive treatment on focal cerebral infarction. Hypertension 1992, 19:713-716.

21. Watson BD, Dietrich WD, Busto R, Wachtel MS, Ginsberg MD: Induction of reproducible brain infarction by photochemically initiated thrombosis. Ann Neurol 1985, 17:497-504.

22. Prado R, Watson BD, Zhao W, Yao H, Busto R, Dietrich WD, Ginsberg MD: L-arginine does not improve cortical perfusion or histopathological outcome in spontaneously hypertensive rats subjected to distal middle cerebral artery photothrombotic occlusion. I Cereb Blood Flow Metab 1996, 16:612-22.

23. Yao H, Ibayashi S, Sugimori H, Fujii K, Fujishima M: Simplified model of krypton laser-induced thrombotic distal middle cerebral artery occlusion in spontaneously hypertensive rats. Stroke 1996, 27:333-336.

24. Cai H, Yao H, Ibayashi S, Uchimura H, Fujishima M: Photothrombotic middle cerebral artery occlusion in spontaneously hypertensive rats: influence of substrain, gender, and distal middle cerebral artery patterns on infarct size. Stroke 1998, 29:1982-1986

25. Ogata J, Fujishima M, Morotomi Y, Omae T: Cerebral infarction following bilateral carotid artery ligation in normotensive and spontaneously hypertensive rats: a pathological study. Stroke 1976, 7:54-60

26. Watson BD, Prado R, Veloso A, Brunschwig JP, Dietrich WD: Cerebral blood flow restoration and reperfusion injury after ultraviolet laser-facilitated middle cerebral artery recanalization in rat thrombotic stroke. Stroke 2002, 33:428-434.

27. Yao H, Nabika T: Characterizing photothrombotic distal middle cerebral artery occlusion and YAG laser-induced reperfusion model in the Izumo strain of spontaneously hypertensive rats. Cell Mol Neurobiol 2011, 31:57-63.

28. Koizumi J, Yoshida Y, Nakazawa T, Ooneda G: Experimental studies of ischemic brain edema. l: a new experimental model of cerebral embolism in rats in which recirculation can be introdued in the ischemic area. Jpn J Stroke 1986, 8:1-8

29. Longa EZ, Weinstein PR, Carlson S, Cummins R: Reversible middle cerebral artery occlusion without craniectomy in rats. Stroke 1989, 20:84-91.

30. Howells DW, Porritt MJ, Rewell SS, O'Collins V, Sena ES, van der Worp HB, Traystman RJ, Macleod MR: Different strokes for different folks: the rich diversity of animal models of focal cerebral ischemia. J Cereb Blood Flow Metab 2010, 30:1412-1431.

31. Kawamura S, Li Y, Shirasawa M, Yasui N, Fukasawa H: Protective effect of nilvadipine on focal cerebral ischemia in spontaneously hypertensive rats. Neurol Med Chir (Tokyo) 1996, 36:151-155.

32. Alkayed NJ, Harukuni I, Kimes AS, London ED, Traystman RJ, Hurn PD: Gender-linked brain injury in experimental stroke. Stroke 1998 , 29:159-165.

33. Lakhan SE, Kirchgessner A, Hofer M: Inflammatory mechanisms in ischemic stroke: therapeutic approaches. J Trans/ Med 2009, 7:7-97.

34. Kanemitsu H, Nakagomi T, Tamura A, Tsuchiya T, Kono G, Sano K: Differences in the extent of primary ischemic damage between middle cerebral artery coagulation and intraluminal occlusion models. J Cereb Blood Flow Metab 2002, 22:1196-1204.

35. Henning EC, Latour LL, Hallenbeck JM, Warach S: Reperfusion-associated hemorrhagic transformation in SHR rats: evidence of symptomatic parenchymal hematoma. Stroke 2008, 39:3405-3410.

36. Guan W, Kozak A, Fagan SC: Drug repurposing for vascular protection after acute ischemic stroke. Acta Neurochir Suppl 2011, 111:295-298.

37. Yamashita T, Kamiya T, Deguchi K, Inaba T, Zhang H, Shang J, Miyazaki K, Ohtsuka A, Katayama Y, Abe K: Dissociation and protection of the neurovascular unit after thrombolysis and reperfusion in ischemic rat brain. J Cereb Blood Flow Metab 2009, 29:715-725.

38. The National Institute of Neurological Disorders and Stroke rt-PA Stroke Study Group: Tissue plasminogen activator for acute ischemic stroke. N Engl J Med 1995, 333:1581-1587.

39. Hacke W, Kaste M, Bluhmki E, Brozman M, Dávalos A, Guidetti D, Larrue V, Lees KR, Medeghri Z, Machnig T, Schneider D, von Kummer R, Wahlgren N Toni D: ECASS Investigators: Thrombolysis with alteplase 3 to 4.5 hours after acute ischemic stroke. N Engl J Med 2008, 359:1317-1329.

40. Wahlgren N, Ahmed N, Eriksson N, Aichner F, Bluhmki E, Dávalos A, Erilä T, Ford GA, Grond M, Hacke W, Hennerici MG, Kaste M, Köhrmann M, Larrue V, Lees KR, Machnig T, Roine RO, Toni D, Vanhooren G: Safe Implementation of Thrombolysis in Stroke-MOnitoring STudy Investigators: Multivariable analysis of outcome predictors and adjustment of main outcome results to baseline data profile in randomized controlled trials: Safe 
Implementation of Thrombolysis in Stroke-MOnitoring STudy (SITS-MOST). Stroke 2008, 39:3316-3322.

41. Asahi M, Asahi K, Wang X, Lo EH: Reduction of tissue plasminogen activator-induced hemorrhage and brain injury by free radical spin trapping after embolic focal cerebral ischemia in rats. $J$ Cereb Blood Flow Metab 2000, 20:452-457.

42. Tejima E, Katayama Y, Suzuki Y, Kano T, Lo EH: Hemorrhagic transformation after fibrinolysis with tissue plasminogen activator: evaluation of role of hypertension with rat thromboembolic stroke model. Stroke 2001, 32:1336-1340

43. Sun L, Zhou W, Mueller C, Sommer C, Heiland S, Bauer AT, Marti HH, Veltkamp R: Oxygen therapy reduces secondary hemorrhage after thrombolysis in thromboembolic cerebral ischemia. J Cereb Blood Flow Metab 2010, 30:1651-1660.

44. Fujiwara N, Murata Y, Arai K, Egi Y, Lu J, Wu O, Singhal AB, Lo EH: Combination therapy with normobaric oxygen (NBO) plus thrombolysis in experimental ischemic stroke. BMC Neurosci 2009, 10:79.

45. Astrup J, Symon L, Branston NM, Lassen NA: Cortical evoked potential and extracellular $\mathrm{K}^{+}$and $\mathrm{H}^{+}$at critical levels of brain ischemia. Stroke 1977 8:51-57.

46. Zhao W, Belayev L, Ginsberg MD: Transient middle cerebral artery occlusion by intraluminal suture: II. Neurological deficits, and pixel-based correlation of histopathology with local blood flow and glucose utilization. J Cereb Blood Flow Metab 1997, 17:1281-1290.

47. Hossmann KA: Viability thresholds and the penumbra of focal ischemia. Ann Neurol 1994, 36:557-565.

48. Sun PZ, Zhou J, Sun W, Huang J, van Zijl PC: Detection of the ischemic penumbra using pH-weighted MRI. J Cereb Blood Flow Metab 2007, 27:1129-1136.

49. Heiss WD: The ischemic penumbra: correlates in imaging and implications for treatment of ischemic stroke. The Johann Jacob Wepfer award 2011. Cerebrovasc Dis 2011, 32:307-320

50. Campbell BC, Purushotham A, Christensen S, Desmond PM, Nagakane Y, Parsons MW, Lansberg MG, Mlynash M, Straka M, De Silva DA, Olivot JM, Bammer R, Albers GW, Donnan GA, Davis SM: The infarct core is well represented by the acute diffusion lesion: sustained reversal is infrequent. J Cereb Blood Flow Metab 2012, 32:50-56.

51. Jones SC, Kharlamov A, Yanovski B, Kim DK, Easley KA, Yushmanov VE, Ziolko SK, Boada FE: Stroke onset time using sodium MRI in rat focal cerebral ischemia. Stroke 2006, 37:883-888.

52. De Silva DA, Brekenfeld C, Ebinger M, Christensen S, Barber PA, Butcher KS, Levi CR, Parsons MW, Bladin CF, Donnan GA, Davis SM: Echoplanar Imaging Thrombolytic Evaluation Trial (EPITHET) Investigators: The benefits of intravenous thrombolysis relate to the site of baseline arterial occlusion in the Echoplanar Imaging Thrombolytic Evaluation Trial (EPITHET). Stroke 2010, 41:295-299.

53. Letourneur A, Roussel S, Toutain J, Bernaudin M, Touzani O: Impact of genetic and renovascular chronic arterial hypertension on the acute spatiotemporal evolution of the ischemic penumbra: a sequential study with MRI in the rat. J Cereb Blood Flow Metab 2011, 31:504-513.

54. Yao H, Okada Y, Ibayashi S: Therapeutic time window for YAG laser-induced reperfusion of thrombotic stroke in hypertensive rats. Neuroreport 2002, 13:1005-1008

55. Liszczak TM, Hedley-Whyte ET, Adams JF, Han DH, Kolluri VS, Vacanti FX, Heros RC, Zervas NT: Limitations of tetrazolium salts in delineating infarcted brain. Acta Neuropathol 1984, 65:150-157.

56. Zille M, Farr TD, Przesdzing I, Müller J, Sommer C, Dirnagl U, Wunder A: Visualizing cell death in experimental focal cerebral ischemia: promises, problems, and perspectives. J Cereb Blood Flow Metab. 2012, 32:213-231

57. Yao H, Yoshii N, Akira T, Nakahara T: Reperfusion-induced temporary appearance of therapeutic window in penumbra after $2 \mathrm{~h}$ of photothrombotic middle cerebral artery occlusion in rats. J Cereb Blood Flow Metab 2009, 29:565-574

58. Yao H, Takasawa R, Fukuda K, Shiokawa D, Sadanaga-Akiyoshi F, Ibayashi S, Tanuma S, Uchimura H: DNA fragmentation in ischemic core and penumbra in focal cerebral ischemia in rats. Brain Res Mol Brain Res 2001, 91:112-118.

59. Yao H, Nakahara T, Nakagawa N, Hashimoto K, Kuroki T: Regional and temporal changes in proteomic profile after middle cerebral artery occlusion with or without reperfusion in rats. Neurochem Res 2009, 34:1999-2007.

60. Braeuninger S, Kleinschnitz C: Rodent models of focal cerebral ischemia: procedural pitfalls and translational problems. Exp Trans/ Stroke Med 2009, 25:1-8.

61. Dirnagl U, Pulsinelli W: Autoregulation of cerebral blood flow in experimental focal brain ischemia. J Cereb Blood Flow Metab 1990, 10:327-336.

62. Zhu CZ, Auer RN: Graded hypotension and MCA occlusion duration: effect in transient focal ischemia. J Cereb Blood Flow Metab 1995, 15:980-988.

63. Zausinger S, Baethmann A, Schmid-Elsaesser R: Anesthetic methods in rats determine outcome after experimental focal cerebral ischemia: mechanical ventilation is required to obtain controlled experimental conditions. Brain Res Brain Res Protoc 2002, 9:112-121.

64. Yao H, Matsumoto T, Hirano M, Kuroki T, Tsutsumi T, Uchimura H, Nakamura K, Nakahara T, Fujishima M: Involvement of brain stem noradrenergic neurons in the development of hypertension in spontaneously hypertensive rats. Neurochem Res 1989, 14:75-79.

65. Mori S, Kato M, Fujishima M: Impaired maze learning and cerebral glucose utilization in aged hypertensive rats. Hypertension 1995, 25:545-553.

66. Yao H, Cui ZH, Masuda J, Nabika T: Congenic removal of a QTL for blood pressure attenuates infarct size produced by middle cerebral artery occlusion in hypertensive rats. Physiol Genomics 2007, 30:69-73.

67. DeBow SB, Clark DL, MacLellan CL, Colbourne F: Incomplete assessment of experimental cytoprotectants in rodent ischemia studies. Can J Neurol Sci. 2003, 30:368-374.

68. Hurn PD, Vannucci SJ, Hagberg H: Adult or perinatal brain injury: does sex matter? Stroke 2005, 36:193-195.

69. Fukuda K, Yao H, Ibayashi S, Nakahara T, Uchimura H, Fujishima M: Ovariectomy exacerbates and estrogen replacement attenuates photothrombotic focal ischemic brain injury in rats. Stroke 2000 31:155-160.

70. Macleod MR, O'Collins T, Howells DW, Donnan GA: Pooling of animal experimental data reveals influence of study design and publication bias. Stroke 2004, 35:1203-1208.

71. Stroke Therapy Academic Industry Roundtable (STAIR): Recommendations for Standards Regarding Preclinical Neuroprotective and Restorative Drug Development. Stroke 1999, 30:2752-2758.

72. Macleod MR, O'Collins T, Horky LL, Howells DW, Donnan GA: Systematic review and metaanalysis of the efficacy of FK506 in experimental stroke. J Cereb Blood Flow Metab 2005, 25:713-721.

73. O'Collins VE, Macleod MR, Donnan GA, Horky LL, van der Worp BH, Howells DW: 1,026 experimental treatments in acute stroke. Ann Neurol 2006, 59:467-477.

74. Sandset EC, Bath PM, Boysen G, Jatuzis D, Körv J, Lüders S, Murray GD, Richter PS, Roine RO, Terént A, Thijs V: Berge E; SCAST Study Group: The angiotensin-receptor blocker candesartan for treatment of acute stroke (SCAST): a randomised, placebo-controlled, double-blind trial. Lancet 2011, 377:741-750

75. Kozak W, Kozak A, Johnson MH, Elewa HF, Fagan SC: Vascular protection with candesartan after experimental acute stroke in hypertensive rats: a dose-response study. J Pharmacol Exp Ther 2008, 326:773-782.

76. McCabe C, Gallagher L, Gsell W, Graham D, Dominiczak AF, Macrae IM: Differences in the evolution of the ischemic penumbra in stroke-prone spontaneously hypertensive and Wistar-Kyoto rats. Stroke. 2009, 40:3864-3868

77. Dijkhuizen RM, Asahi M, Wu O, Rosen BR, Lo EH: Rapid breakdown of microvascular barriers and subsequent hemorrhagic transformation after delayed recombinant tissue plasminogen activator treatment in a rat embolic stroke model. Stroke. 2002, 33:2100-2104.

78. Riyamongkol P, Zhao W, Liu Y, Belayev L, Busto R, Ginsberg MD: Automated registration of laser Doppler perfusion images by an adaptive correlation approach: application to focal cerebral ischemia in the rat. J Neurosci Methods 2002, 122:79-90

79. Ren Y, Hashimoto M, Pulsinelli WA, Nowak TS Jr: Hypothermic protection in rat focal ischemia models: strain differences and relevance to "reperfusion injury". J Cereb Blood Flow Metab 2004, 24:42-53.

80. Serikawa T, Mashimo T, Takizawa A, Okajima R, Maedomari N, Kumafuji K, Tagami F, Neoda Y, Otsuki M, Nakanishi S, Yamasaki K, Voigt B, Kuramoto T: National BioResource Project-Rat and related activities. Exp Anim 2009, 58:333-341. 
81. Mashimo T, Voigt B, Tsurumi T, Naoi K, Nakanishi S, Yamasaki K, Kuramoto T, Serikawa T: A set of highly informative rat simple sequence length polymorphism (SSLP) markers and genetically defined rat strains. BMC Genet 2006, 7:19.

82. Jeffs B, Clark JS, Anderson NH, Gratton J, Brosnan MJ, Gauguier D, Reid JL, Macrae IM, Dominiczak AF: Sensitivity to cerebral ischaemic insult in a rat model of stroke is determined by a single genetic locus. Nat Genet 1997, 16:364-367.

83. Mashimo T, Takizawa A, Voigt B, Yoshimi K, Hiai H, Kuramoto T, Serikawa T: Generation of knockout rats with $X$-linked severe combined immunodeficiency (X-SCID) using zinc-finger nucleases. PLoS One 2010, 5:e8870.

84. Rubattu S, Gigante B, Stanzione R, De Paolis P, Tarasi D, Volpe M: In the search for stroke genes: a long and winding road. Am J Hypertens 2004, 17:197-202

85. Carr FJ, McBride MW, Carswell HV, Graham D, Strahorn P, Clark JS, Charchar FJ, Dominiczak AF: Genetic aspects of stroke: human and experimental studies. J Cereb Blood Flow Metab 2002, 22:767-773.

86. Graham D, McBride MW, Gaasenbeek M, Gilday K, Beattie E, Miller WH, McClure JD, Polke JM, Montezano A, Touyz RM, Dominiczak AF: Candidate genes that determine response to salt in the stroke-prone spontaneously hypertensive rat: congenic analysis. Hypertension 2007, 50:1134-1141.

87. Aitman TJ, Glazier AM, Wallace CA, Cooper LD, Norsworthy PJ, Wahid FN, Al-Majali KM, Trembling PM, Mann CJ, Shoulders CC, Graf D, St Lezin E, Kurtz TW, Kren V, Pravenec M, Ibrahimi A, Abumrad NA, Stanton LW, Scott J: Identification of $\mathrm{Cd} 36$ (Fat) as an insulin-resistance gene causing defective fatty acid and glucose metabolism in hypertensive rats. Nat Genet 1999, 21:76-83.

doi:10.1186/1479-5876-10-139

Cite this article as: Yao and Nabika: Standards and pitfalls of focal ischemia models in spontaneously hypertensive rats: With a systematic review of recent articles. Journal of Translational Medicine 2012 10:139.

\section{Submit your next manuscript to BioMed Central and take full advantage of:}

- Convenient online submission

- Thorough peer review

- No space constraints or color figure charges

- Immediate publication on acceptance

- Inclusion in PubMed, CAS, Scopus and Google Scholar

- Research which is freely available for redistribution 